\title{
REVIEW \\ Abdominal functional electrical stimulation to improve respiratory function after spinal cord injury: a systematic review and meta-analysis
}

\author{
This article has been corrected since Advance Online Publication and a corrigendum is also printed in this issue.
}

\author{
EJ McCaughey ${ }^{1}$, RJ Borotkanics ${ }^{1,2}, \mathrm{H} \mathrm{Gollee}^{3,4}, \mathrm{RJ}$ Folz $^{5}$ and AJ McLachlan ${ }^{6}$
}

Objectives: Abdominal functional electrical stimulation (abdominal FES) is the application of a train of electrical pulses to the abdominal muscles, causing them to contract. Abdominal FES has been used as a neuroprosthesis to acutely augment respiratory function and as a rehabilitation tool to achieve a chronic increase in respiratory function after abdominal FES training, primarily focusing on patients with spinal cord injury (SCI). This study aimed to review the evidence surrounding the use of abdominal FES to improve respiratory function in both an acute and chronic manner after $\mathrm{SCl}$.

Settings: A systematic search was performed on PubMed, with studies included if they applied abdominal FES to improve respiratory function in patients with $\mathrm{SCl}$.

Methods: Fourteen studies met the inclusion criteria (10 acute and 4 chronic). Low participant numbers and heterogeneity across studies reduced the power of the meta-analysis. Despite this, abdominal FES was found to cause a significant acute improvement in cough peak flow, whereas forced exhaled volume in $1 \mathrm{~s}$ approached significance. A significant chronic increase in unassisted vital capacity, forced vital capacity and peak expiratory flow was found after abdominal FES training compared with baseline.

Conclusions: This systematic review suggests that abdominal FES is an effective technique for improving respiratory function in both an acute and chronic manner after SCl. However, further randomised controlled trials, with larger participant numbers and standardised protocols, are needed to fully establish the clinical efficacy of this technique.

Spinal Cord (2016) 54, 628-639; doi:10.1038/sc.2016.31; published online 12 April 2016

\section{INTRODUCTION}

Functional electrical stimulation (FES) is the application of a train of electrical pulses to a motor nerve, causing the associated muscle to contract. ${ }^{1}$ The application of FES to the abdominal muscles is called abdominal functional electrical stimulation (abdominal FES). For patients with impaired ventilatory respiration, for example, due to paralysis affecting the major respiratory muscles, abdominal FES can be used to activate paralysed abdominal muscles, which may achieve an acute (immediate and temporary) improvement in respiratory function. ${ }^{2-9}$ The repeated application of abdominal FES, termed abdominal FES training, has been hypothesised to increase abdominal muscle mass and tone, placing the diaphragm in a more efficient position for respiration. ${ }^{10}$ Abdominal FES training may improve the unassisted respiratory function in patients with impaired respiration.

Although there have been a limited number of studies investigating the use of abdominal FES to improve respiratory function in patients with stroke, ${ }^{11}$ traumatic brain injury ${ }^{12}$ and chronic obstructive pulmonary disease, ${ }^{13}$ the vast majority of clinical investigations of abdominal FES have focused on patients with spinal cord injury
(SCI). ${ }^{10,14,15}$ These patients commonly have paralysis or impaired function of the major respiratory muscles: namely, the diaphragm, intercostal muscles and abdominal muscles. As a result, SCI is associated with reduced levels of respiratory function compared with the able-bodied population, with tetraplegia resulting in an $\sim 50 \%$ reduction in respiratory function. ${ }^{16}$ Reduced respiratory function leaves people with an SCI unable to clear their airways through cough, often leading to respiratory complications such as atelectasis, pneumonia and ventilatory failure, ${ }^{17}$ being a primary cause of morbidity and mortality for this population. ${ }^{18}$ An improvement in respiratory function has been shown to positively correlate with a reduction in respiratory complications. ${ }^{14}$ If abdominal FES is effective in improving respiratory function, then this could have a significant impact on morbidity and mortality for the SCI population.

Despite the positive results achieved in individual abdominal FES studies in SCI, there has been no pooled analysis of the effect of abdominal FES on respiratory function. A systematic review and meta-analysis can be used to pool results from different studies, thereby enhancing the precision of estimates of treatment effects. ${ }^{19}$

${ }^{1}$ Centre for Health Systems and Safety Research, Australian Institute for Health Innovation, Macquarie University, North Ryde, New South Wales, Australia; ${ }^{2}$ Bloomberg School of Public Health, John Hopkins University, MD, USA; ${ }^{3}$ School of Engineering, University of Glasgow, Scotland, UK; ${ }^{4}$ Scottish Centre for Innovation in Spinal Cord Injury, Glasgow, Scotland, UK; ${ }^{5}$ School of Medicine, Case Western Reserve University, Cleveland, OH, USA and ${ }^{6}$ Liberate Medical LLC, Crestwood, KY, USA Correspondence: Dr EJ McCaughey, Australian Institute for Health Innovation, Macquarie University, Sydney, New South Wales 2109 , Australia.

Email: euan.mccaughey@mq.edu.au

Received 8 November 2015; revised 31 January 2016; accepted 3 February 2016; published online 12 April 2016 
The aim of this systematic review and meta-analysis was to identify whether abdominal FES is an effective intervention to improve respiratory function in both an acute and chronic manner after SCI.

\section{METHODS}

\section{Literature search}

Two authors (EJM and RJF) independently searched PubMed on the 23 December 2014 for peer-reviewed articles that investigated the effect of abdominal FES on respiratory function. The keywords used for the search were either abdominal or abdominal muscles, and electrical stimulation or functional electrical stimulation or neuromuscular electrical stimulation or muscle stimulation or stimulation, and breathing or respiratory or respiration or cough or spirometry or tidal volume or inspiratory or expiratory. Reference lists from identified articles were also hand searched for articles not returned in the initial search.

\section{Study selection and eligibility criteria}

Articles were included if they applied abdominal FES and measured respiratory function. Articles were excluded if they were as follows: not in English; duplicates (or where participants in studies were not independent of a previous publication); experiments in animals; single case reports; meeting abstracts; and editorials or reviews. Furthermore, to remove a significant source of heterogeneity, two studies that met the aforementioned inclusion criteria but included patients who did not have SCI were excluded from the meta-analysis. ${ }^{11,12}$

Initially, all abstracts and titles were independently reviewed (EJM and RJF) and sorted based on the predefined inclusion criteria. The full text of the studies that match these criteria was then independently reviewed (EJM and RJF) and once again sorted based on the predefined inclusion criteria. Both reviewers held a meeting to confirm the articles to be included in the review. In cases of disagreement, a third reviewer (AJM) made the final decision. Single-case studies were noted and used for informational purposes but were excluded from the analysis, so as to avoid the redundant reporting of findings. The search protocol, which was registered on the PROSPERO registry (registration number: CRD42015024218), is summarised in Figure 1.

\section{Measures of respiratory function}

A number of measures of respiratory function were used to measure the efficacy of abdominal FES in this analysis. Cough peak flow (CPF) is the maximum rate at which air can be exhaled from the lungs during a cough, whereas peak expiratory flow (PEF) is the maximum rate at which air can be exhaled from the lungs when measuring from total lung capacity and exhaling as forcefully and as quickly as possible. As both CPF and PEF are measured during exhalation, they can be used to indicate the strength of the expiratory muscles. Maximum expiratory pressure (MEP) is the maximum pressure generated at the mouth when exhaling from total lung capacity as forcefully as possible against an occluded airway and hence can also be used to measure the strength of the expiratory muscles. Vital capacity $\left(V_{\mathrm{C}}\right)$, the total volume of air exhaled after inhaling to total lung capacity and exhaling passively, and forced vital capacity (FVC), the total volume of air exhaled after inhaling to total lung capacity and exhaling as forcefully as possible, can be used to evaluate the strength of the inspiratory muscles $\left(\mathrm{V}_{\mathrm{C}}\right.$ and $\left.\mathrm{FVC}\right)$ and the expiratory muscles (FVC). These measures are closely related, with the only difference being the lower level of effort exerted during $\mathrm{V}_{\mathrm{C}}$ exhalation. ${ }^{20}$ When measuring FVC, forced exhaled volume in $1 \mathrm{~s}\left(\mathrm{FEV}_{1}\right)$, which is the volume of air that can be exhaled from the lungs in the first second of a forced exhalation, can also be measured to provide an indication of the strength of the expiratory muscles. Finally, gastric $\left(P_{\text {ga }}\right)$ and oesophageal pressure $\left(P_{\text {es }}\right)$ can be used to measure the pleural pressures achieved during contraction of the expiratory muscles, providing another indication of the strength of the expiratory muscles.

\section{Analysis}

Authors of studies that met the inclusion criteria but where a lack of information in the manuscript meant that the study could not be included in the initial meta-analysis were contacted and asked to provide additional information. ${ }^{2,10,15}$ McLachlan et al. ${ }^{10}$ also provided acute data that were not published in the original manuscript.

Studies that met the inclusion criteria were classified into two broad categories, namely, acute and chronic. Acute studies compared respiratory function before and during abdominal FES. These studies applied a self-control study design. Chronic studies measured the chronic effect of abdominal FES training. These studies applied either a self-control (randomised crossover) study design or a randomised controlled trial (RCT) approach, with all chronic

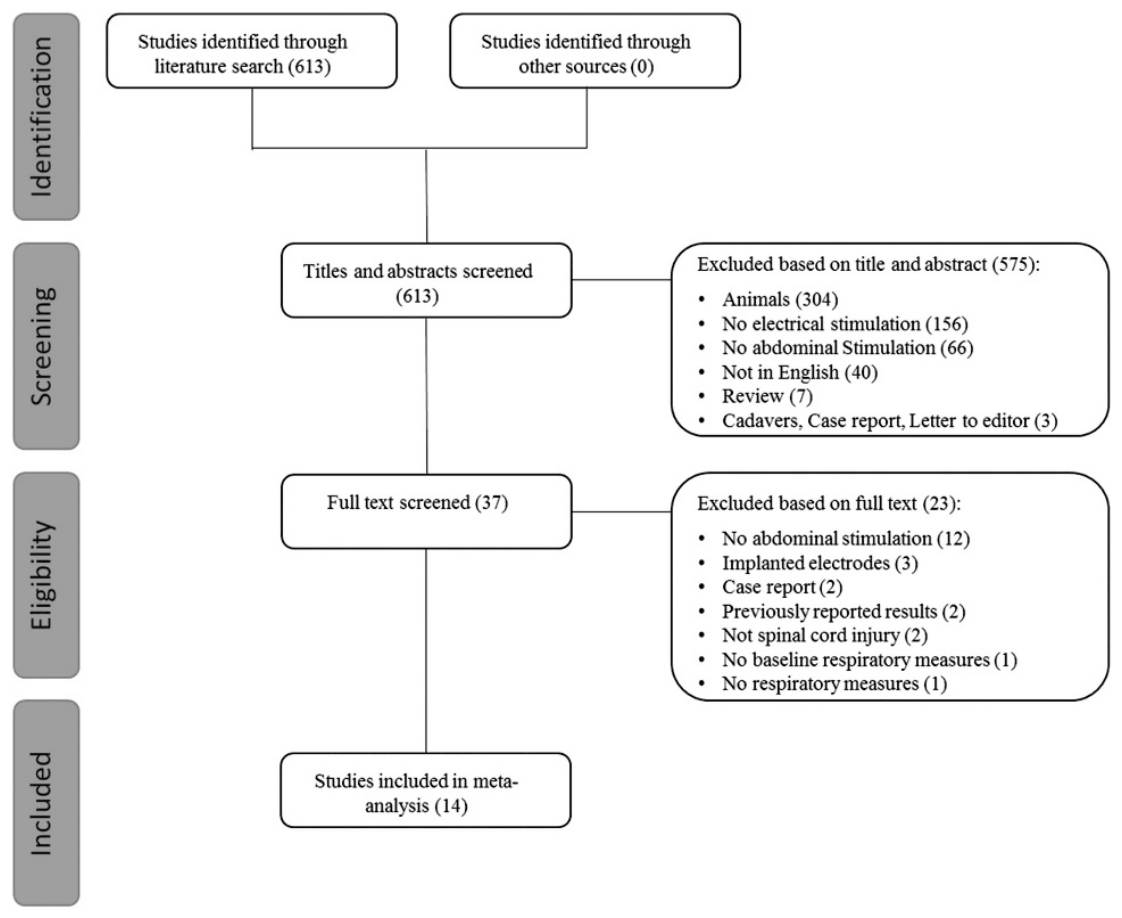

Figure 1 PRISMA flow diagram of search protocol. A full color version of this figure is available at the Spinal Cord journal online. 


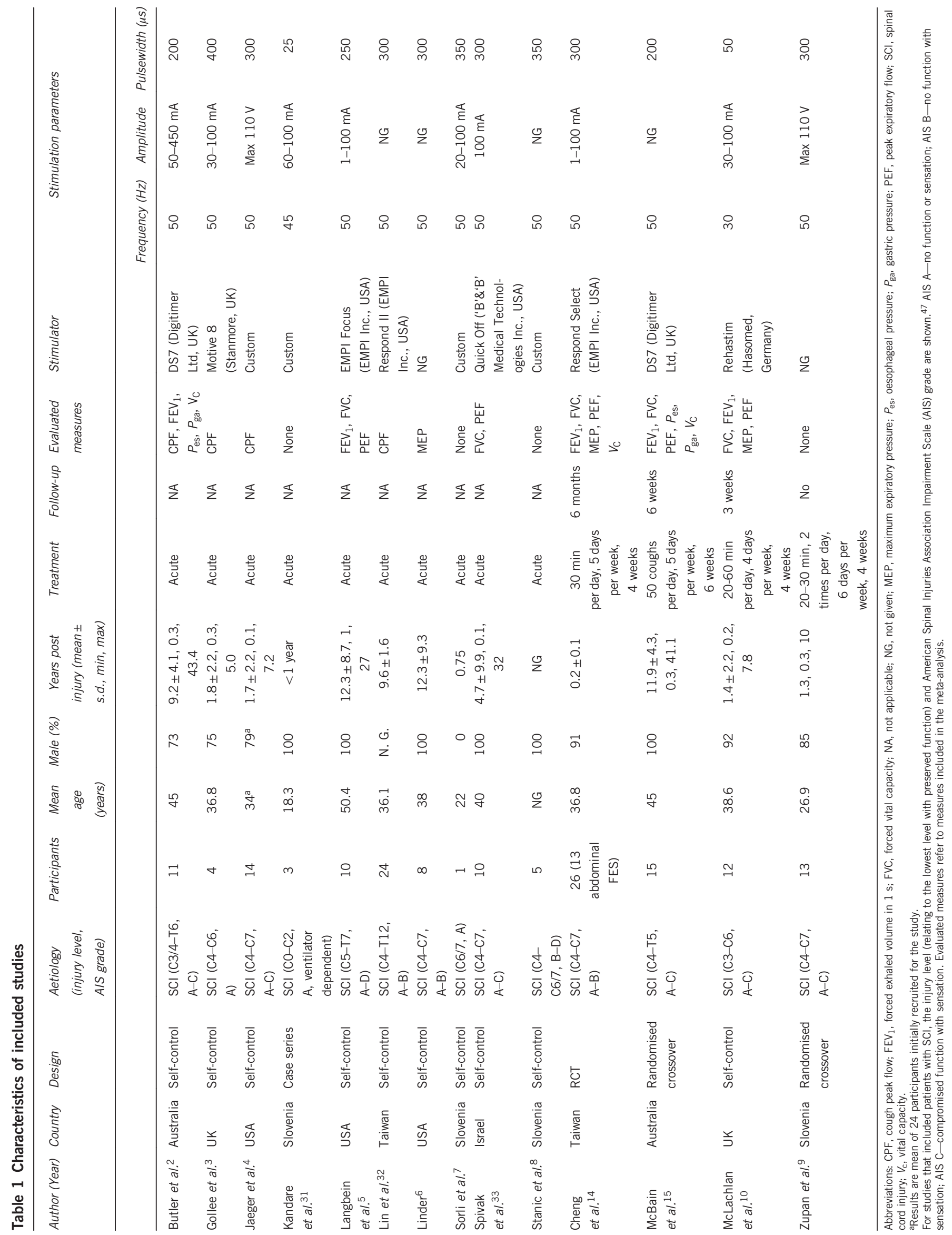


a

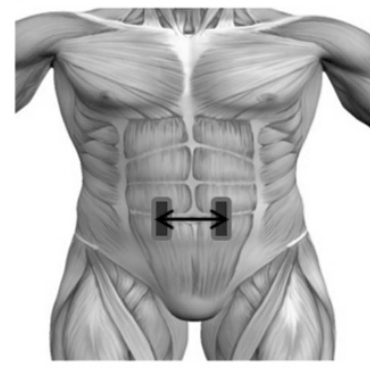

Jaeger et al. ${ }^{4}$

d

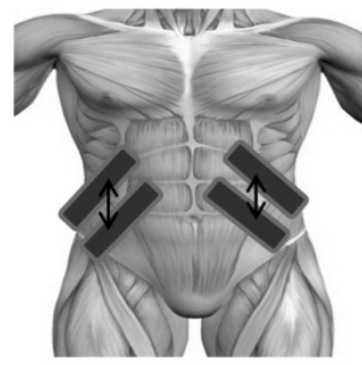

Butler et al., ${ }^{2}$ McBain et al. ${ }^{15}$ b

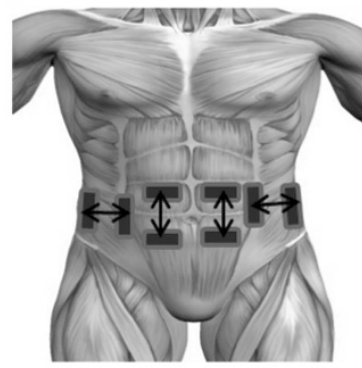

Kandare et al. ${ }^{31}$

e

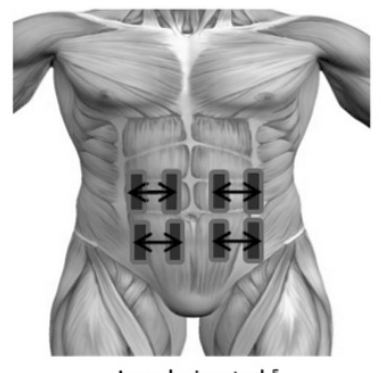

Langbein et al. ${ }^{5}$

g

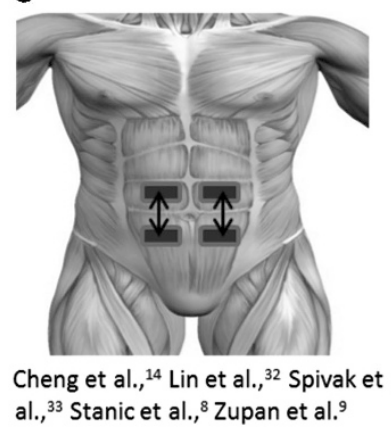

C

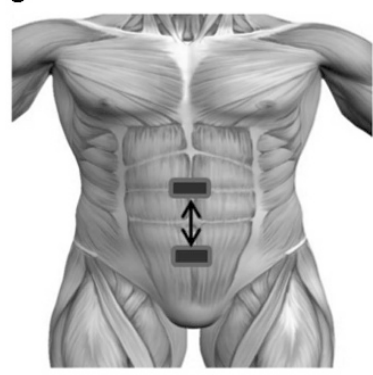

Sorli et al. ${ }^{7}$

f

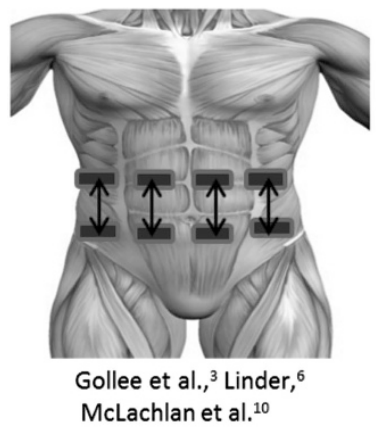

McLachlan et al. ${ }^{10}$

Figure 2 Electrode positions used to deliver abdominal FES. A full color version of this figure is available at the Spinal Cord journal online.

studies included in the meta-analysis applying a three-phase approach. The first phase was the baseline period, during which time one or more baseline measures of respiratory function were recorded (single time point to 6 weeks). The second phase was the training period, where abdominal FES was applied over a defined time period (4-6 weeks). In the RCT, a matched control group received no intervention during the training period. During the third phase (withdrawal), respiratory function was measured immediately after the conclusion of training, with participants followed up at time points varying between 3 weeks and 6 months.

For both acute and chronic studies, analyses were carried out using either fixed effects models, using the inverse of the variance approach, or random effects models, using the DerSimonian and Laird approach. ${ }^{21}$ Model choice was determined by the between-study heterogeneity of pooled results, which was quantified and assessed using the $I^{2}$ statistic. ${ }^{22,23}$ Where models exhibited low heterogeneity $\left(I^{2}<0.3\right)$, pooled models were analysed using the standard inverse of the variance weighting approach, whereas models exhibiting moderate-to-high heterogeneity $\left(I^{2}>0.3\right)$ were analysed using the DerSimonian and Laird approach. ${ }^{21}$

Because of differences in baseline function between studies, estimates of the effect were made using the standardised mean difference (SMD) approach, applying Glass's $\Delta .{ }^{24}$ This method is preferred where the intervention may potentially alter observed variability and is less susceptible to small sample bias compared with other SMD techniques.
Multiple models were applied to compare time points in the self-control chronic studies, with similar analyses applied to RCTs at equal time points. The reason for this stratified approach is twofold. First, standard approaches to meta-analysis of self-control study designs are continuing to mature, with no standard for such a design. Furthermore, such study designs necessitate controlling or accounting for the natural recovery in respiratory function that occurs in the period immediately following an SCI. ${ }^{25,26}$ Therefore, it was decided that such stratified analysis using the conservative Glass's $\Delta$ was an acceptable methodological compromise considering the present state of the art.

A descriptive approach was used to analyse trends observed in the chronic studies, with data normalised based on minimum within-study values for each measure of respiratory function. This restricted approach was employed because of the variability in baseline measures across studies and the fact that these studies did not measure respiratory function across consistent time points nor conduct the same number of tests across these time points.

Publication bias was assessed using the Begg and Mazumdar test and the Eggar approach..$^{27,28}$ For all statistical tests, a $P$-value of $<0.05$ was considered significant. All statistical analyses were carried out using STATA v. 14.0 (STATA Corp, College Station, TX, USA) and associated updates..$^{29,30}$

\section{RESULTS}

Fourteen studies that investigated the effect of abdominal FES on respiratory function in patients with SCI were included in this review. 
Ten studies investigated the acute effect of abdominal FES, ${ }^{2-8,31-33}$ whereas four studies investigated the chronic effect of abdominal FES training. ${ }^{9}, 10,14,15$

\section{Participants}

The 14 studies included a total of 141 participants (mean age 37.8 years, $90.1 \%$ male), with 128 (mean age 37.7 years, $89.2 \%$ male) receiving abdominal FES and 13 (mean age 38.8 years, $76.9 \%$ male) acting as controls (Table 1). None of the patients had been exposed to abdominal FES before participation in any of the studies. However, all patients were receiving standard SCI rehabilitation, which was likely to have included exercises aimed at strengthening the abdominal muscles.

\section{Protocols}

The stimulation parameters used in each study are shown in Table 1, whereas a graphical representation of the electrode positions used in each study is provided in Figure 2. The median maximum amplitude was $100 \mathrm{~mA}$ (range 100-450 mA), the mean pulsewidth (pulse duration) was $259 \mu$ s (range 25-400 $\mu \mathrm{s}$ ) and almost all studies used a stimulation frequency of $50 \mathrm{~Hz}$ (Table 1). There was a lack of homogeneity in electrode position, with a range of positions used to stimulate either or both of the rectus abdominis and external oblique muscles (Figure 2).

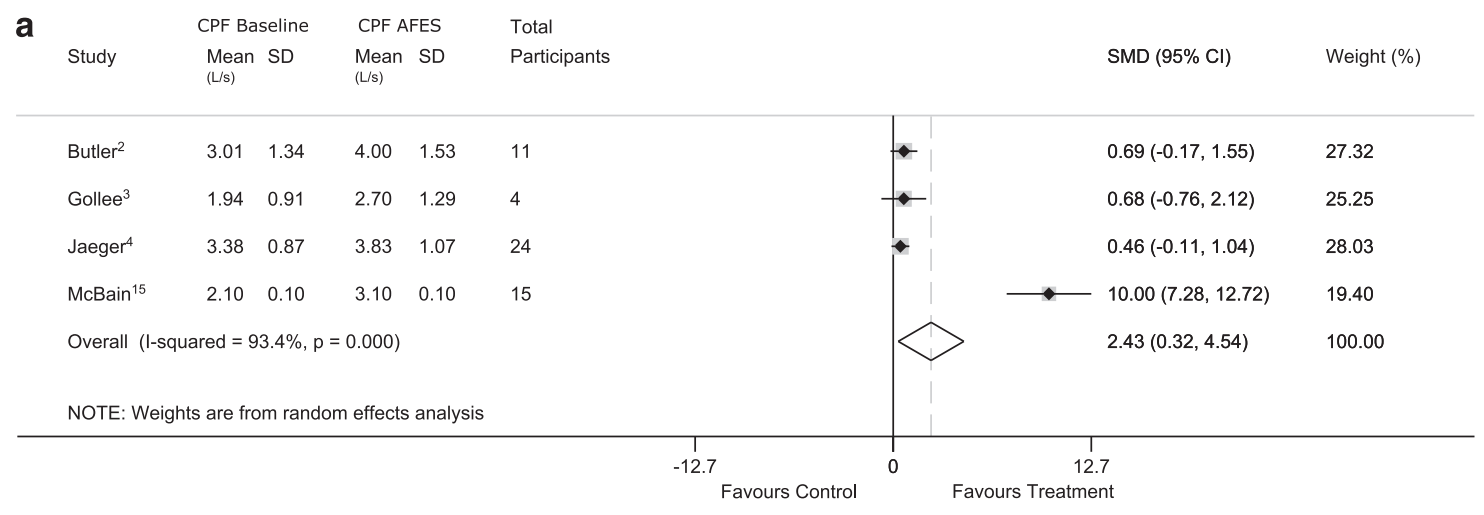

b

\begin{tabular}{|c|c|c|c|c|c|c|c|c|}
\hline \multirow[b]{2}{*}{ Study } & \multicolumn{2}{|c|}{ Baseline } & \multicolumn{2}{|c|}{ AFES } & \multicolumn{4}{|l|}{ Total } \\
\hline & $\begin{array}{l}\text { Mean } \\
\left(\mathrm{cmH}_{2} \mathrm{O}\right)\end{array}$ & SD & $\begin{array}{l}\text { Mean } \\
\left(\mathrm{cmH}_{2} \mathrm{O}\right)\end{array}$ & SD & Participants & & $\operatorname{SMD}(95 \% \mathrm{Cl})$ & Weight (\%) \\
\hline \multicolumn{9}{|c|}{ Pga (cough) } \\
\hline Butler $^{2}$ & 19.50 & 6.00 & 57.90 & 7.00 & 11 & $\leftrightarrow$ & $5.89(3.88,7.90)$ & 51.43 \\
\hline McBain 15 & 1.90 & 0.60 & 37.10 & 2.00 & 15 & $\longrightarrow$ & $23.84(17.56,30.13)$ & 48.57 \\
\hline \multicolumn{6}{|c|}{ Subtotal $(I-$ squared $=96.5 \%, p=0.000)$} & $=$ & $14.61(-2.98,32.19)$ & 100.00 \\
\hline \multicolumn{9}{|c|}{ Pes (cough) } \\
\hline Butler $^{2}$ & 31.20 & 8.70 & 56.60 & 10.50 & 11 & $\bullet$ & $2.63(1.47,3.80)$ & 51.31 \\
\hline McBain 15 & 8.90 & 1.10 & 35.40 & 2.70 & 15 & $\rightarrow-$ & $12.85(9.41,16.30)$ & 48.69 \\
\hline \multicolumn{6}{|c|}{ Subtotal $(\mathrm{I}$-squared $=96.7 \%, \mathrm{p}=0.000)$} & & $7.61(-2.40,17.62)$ & 100.00 \\
\hline \multicolumn{9}{|c|}{ NOTE: Weights are from random effects analysis } \\
\hline & & & & & $\begin{array}{c}1 \\
-32.2\end{array}$ & 0 & & \\
\hline
\end{tabular}

C

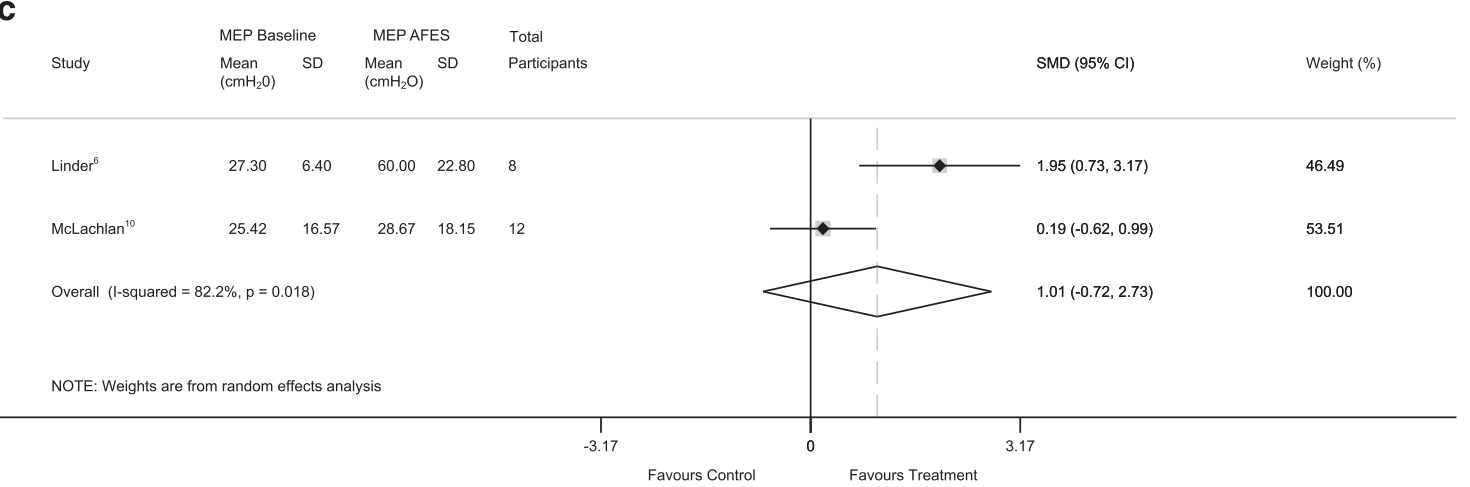

Figure 3 Acute effect of abdominal FES (AFES) on respiratory function. (a) Cough peak flow $(\mathrm{CPF})$, (b) gastric $\left(P_{\text {ga }}\right)$ and oespohageal pressure $\left(P_{\text {es }}\right)$ during cough, (c) maximum expiratory pressure (MEP), (d) forced exhaled volume in $1 \mathrm{~s}$ (FEV $)_{1}$ ), (e) forced vital capacity (FVC), (f) peak expiratory flow (PEF). Standardised mean difference (SMD) is shown on $x$ axis. A positive SMD indicates that the treatment effect favours the intervention, whereas a negative SMD indicates that the treatment effect favours the control (no intervention). For $\mathbf{a}, \mathbf{b}$ and $\mathbf{c}$, pooled analysis uses a random effects model, whereas for $\mathbf{d}$, e and $\mathbf{f}$, pooled analysis uses a fixed effects model. Note: data for McLachlan et al. ${ }^{10}$ were provided by the authors, and data for McBain et al. ${ }^{15}$ only published results to one significant figure. 
d

FEV 1 Baseline FEV $_{1}$ AFES Total

$\begin{array}{ll}\text { Participants } & \text { SMD }(95 \% \mathrm{Cl}) \quad \text { Weight }(\%)\end{array}$

(L)

Mean

(

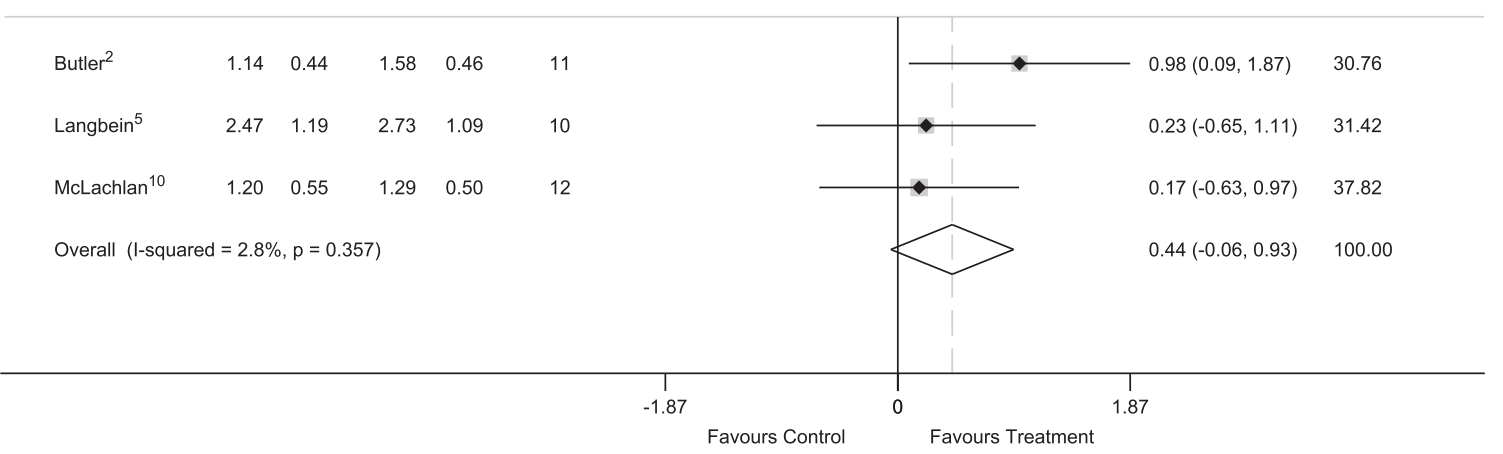

e

FVC Baseline FVC AFES Total

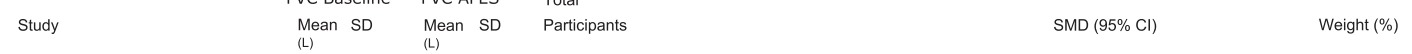

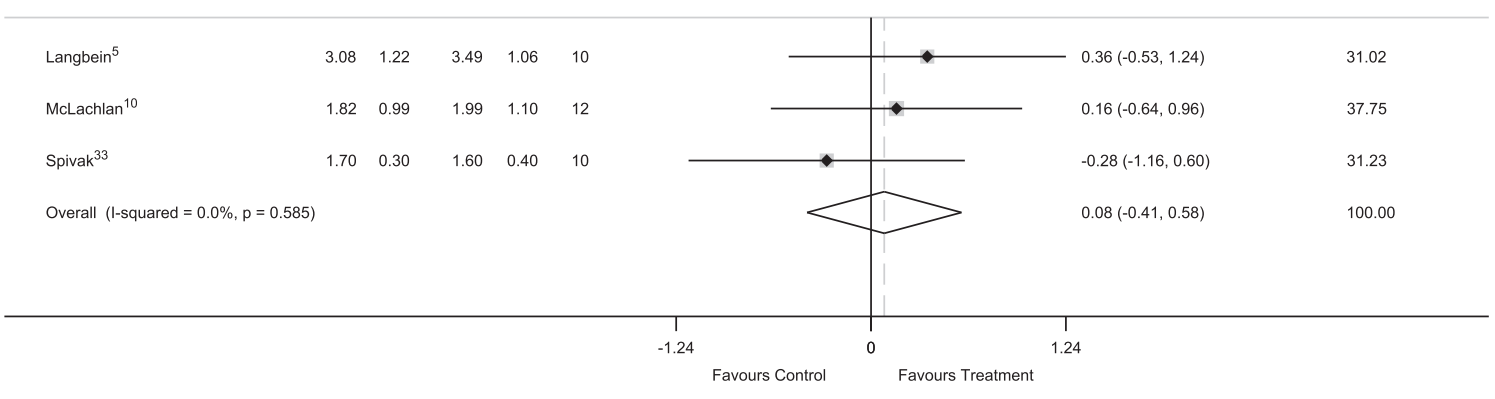

f

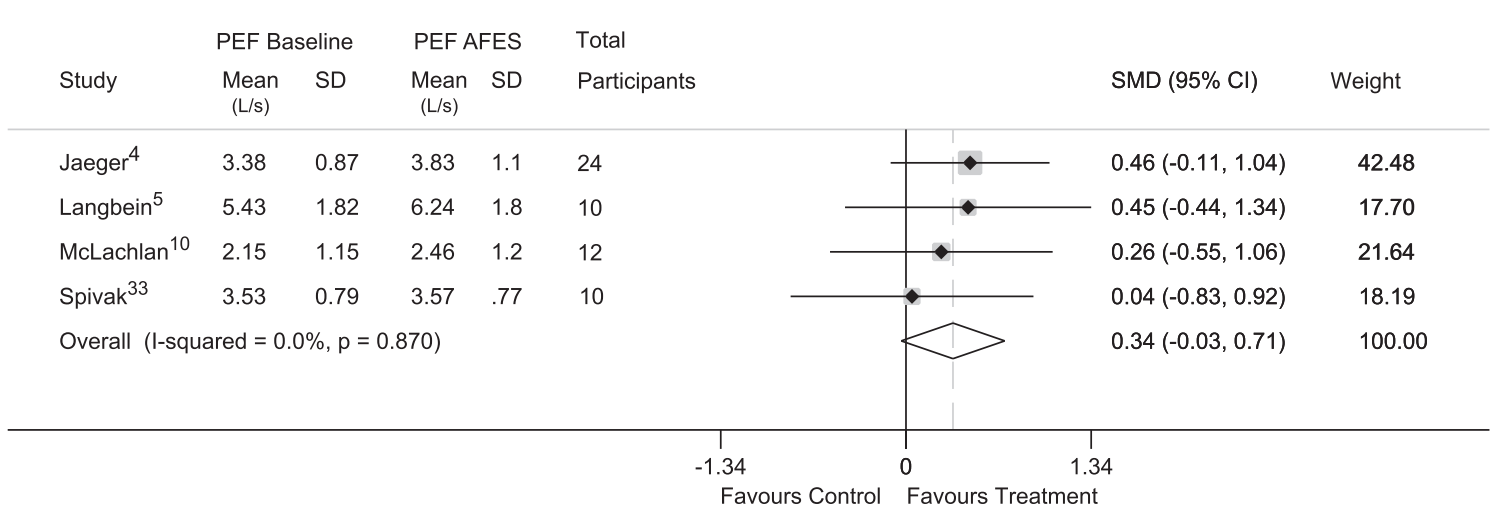

Figure 3 Continued.

\section{RESPIRATORY MEASURES}

\section{Acute effect}

Cough and respiratory muscle strength. The application of abdominal FES was found to lead to a significant increase in CPF (Figure 3a). ${ }^{2-4,15}$ Despite Butler et al. ${ }^{2}$ and McBain et al. ${ }^{15}$ both finding that the application of abdominal FES led to a statistically significant increase in $P_{\mathrm{ga}}$ and $P_{\mathrm{es}}$, significant heterogeneity $\left(I^{2}=96.5 \%\right.$, $P=0.000$ ) between both studies resulted in low confidence in the pooled estimate (Figure $3 \mathrm{~b}$ ). Two studies investigated the acute effect of abdominal FES on MEP.,10 Although Linder et al. ${ }^{6}$ found a significant increase in MEP with abdominal FES, there was low confidence in the pooled estimate (Figure 3c).
Spirometry. Three studies investigated the acute effect of abdominal FES on $\mathrm{FEV}_{1}{ }^{2,5,10}$ Although the study by Butler et al. ${ }^{2}$ was the only one to find a significant effect of abdominal FES on $\mathrm{FEV}_{1}$, the pooled effect approached significance (Figure 3d). Although the three studies that investigated the acute effect of abdominal FES on FVC and PEF displayed homogeneity $\left(I^{2}=0.0 \%, P=0.585\right.$ and $I^{2}=0.0 \%, P=0.870$, respectively), there was no treatment effect (Figures 3 e and f). ${ }^{5,10,33}$

\section{Chronic effect}

Forced vital capacity. A significant increase in FVC was observed after abdominal FES training was applied in three studies $(P=0.043),{ }^{10,14,15}$ whereas Cheng et al. ${ }^{14}$ demonstrated no longitudinal change for a 
Table 2 Longitudinal effect of abdominal FES training on respiratory function between baseline and conclusion of treatment

\begin{tabular}{|c|c|c|c|c|c|c|c|}
\hline Measure & Author & Modality & Participants & $S M D$ & $\mathrm{Cl}$ & Weight & P-value \\
\hline & McBain ${ }^{15}$ & Stim & 15 & 0.213 & -0.507 to 0.933 & 39.82 & \\
\hline \multicolumn{4}{|c|}{ I-V pooled SMD $(R=0.00, \mathrm{df}=2, P=0.593)$} & 0.469 & 0.014 to 0.923 & & 0.043 \\
\hline & Cheng ${ }^{14}$ & Control & 13 & 0.05 & -0.719 to 0.819 & & 0.899 \\
\hline$V_{\mathrm{C}}(\mathrm{I})$ & Cheng ${ }^{14}$ & Stim & 13 & 0.786 & -0.045 to 1.616 & 45.19 & \\
\hline & Cheng ${ }^{14}$ & Control & 13 & 0.12 & -0.650 to 0.890 & & 0.760 \\
\hline \multirow[t]{3}{*}{$\mathrm{FEV}_{1}(\mathrm{I})$} & Cheng $^{14}$ & Stim & 13 & 0.314 & -0.465 to 1.093 & 32.46 & \\
\hline & McBain 15 & Stim & 15 & 0.258 & -0.464 to 0.981 & 37.78 & \\
\hline & McLachlan ${ }^{10}$ & Stim & 12 & 0.35 & -0.463 to 1.163 & 29.77 & \\
\hline \multicolumn{4}{|c|}{ I-V pooled SMD $\left(R^{2}=0.00, \mathrm{df}=1, P=0.986\right)$} & 0.304 & -0.140 to 0.748 & & 0.180 \\
\hline & Cheng14 & Control & 13 & 0 & -0.769 to 0.769 & & 1.000 \\
\hline & Cheng ${ }^{14}$ & Control & 13 & -0.014 & -0.783 to 0.754 & & 0.971 \\
\hline \multirow[t]{2}{*}{$\operatorname{MEP}\left(\mathrm{cmH}_{2} \mathrm{O}\right)$} & Cheng ${ }^{14}$ & Stim & 13 & 0.968 & 0.107 to 1.828 & 46.36 & \\
\hline & McLachlan ${ }^{10}$ & Stim & 12 & 0 & -0.800 to 0.800 & 53.64 & \\
\hline \multicolumn{3}{|c|}{ D-L pooled SMD $\left(P^{2}=61.6, \mathrm{df}=1, P=0.107\right)$} & & 0.47 & -0.478 to 1.418 & & 0.134 \\
\hline & Cheng ${ }^{14}$ & Control & 13 & 0.262 & -0.514 to 1.038 & & 0.508 \\
\hline
\end{tabular}

Abbreviations: $\mathrm{Cl}$, confidence interval; D-L, DerSimonian and Laird approach; $\mathrm{FEV}_{1}$, forced exhaled volume in 1s; FVC, forced vital capacity; I-V, inverse of the variance approach; MEP, maximum expiratory pressure; PEF, peak expiratory flow; SMD, standardised mean difference; $V_{c}$, vital capacity.

Pooled analysis of FVC, $V_{C}, \mathrm{FEV}_{1}$ and PEF uses a fixed effects model, whereas pooled analysis of MEP uses a random effects model. A positive SMD favours the intervention, whereas a negative SMD favours the control.

control group that underwent no training $(P=0.899$; Table 2$)$. All three studies also observed a continued improvement after training (Figure 4a). Cheng et al. ${ }^{14}$ also found a significant difference between the intervention and control group immediately post training $(P=0.020)$ and a trend towards significance 6 months after training $(P=0.128)$. Cheng et al. ${ }^{14}$ and McBain et al. ${ }^{15}$ observed a significant increase in $\mathrm{V}_{\mathrm{C}}$ after abdominal FES training $(P=0.013)$, whereas Cheng et al. demonstrated no longitudinal change for a control group $(P=0.760$; Table 2). After the intervention was removed, Cheng et al. observed an increase in $\mathrm{V}_{\mathrm{C}}$ for both the control and intervention at 3 and 6 months post training; however, McBain et al. observed a decrease in $\mathrm{V}_{\mathrm{C}} 6$ weeks post training (Figure $4 \mathrm{~b}$ ). Cheng et al. also found that the difference between the intervention and control group immediately post and 6 months after training approached significance $(P=0.128$ and $P=0.101$, respectively) (Table 3$)$.

Forced exhaled volume in $1 \mathrm{~s}$. When analysing the three studies that investigated the effect of abdominal FES training on $\mathrm{FEV}_{1}$, the pooled effect was not found to be significant $(P=0.180$; Table 2). Cheng et al. found a continued increase in $\mathrm{FEV}_{1}$ after training, whereas McLachlan and McBain found a decrease in this measure after training (Figure 4c). Cheng et al. also found no difference between the intervention and control group immediately post or 6 months after training $(P=0.353$ and $P=0.894$, respectively; Table 3$)$.

Peak expiratory flow. The pooled treatment effect of the three studies that investigated the effect of abdominal FES training on PEF suggested a significant treatment effect $(P=0.026)$, whereas Cheng et al. demonstrated no longitudinal change for a control group that underwent no training $(P=0.971$; Table 2). Cheng et al. report further increases in PEF after abdominal FES training, whereas McLachlan et al. and McBain et al. report a decrease in PEF at the first time point after training (Figure 4d). Cheng et al. also found a significant difference between the PEF of the intervention and control group immediately after and 6 months after training $(P=0.012$ and $P=0.005$, respectively; Table 3 ).

Maximum expiratory pressure. McLachlan et al. and Cheng et al. investigated the effect of abdominal FES training on MEP, with the pooled effect not found to be significant $(P=0.134$; Table 2). McLachlan et al. observed large variability in MEP, with little change over the training period followed by a large increase during the 3-week post-training period, whereas Cheng et al. observed a substantial increase in MEP between 3 and 6 months post training (Figure 4e). Cheng et al. did observe a statistically significant difference between the MEP of intervention and control participants 6 months after training $(P=0.021)$ and a trend towards significance immediately after training $(P=0.085$; Table 3$)$.

Publication bias. Analysis of publication bias identified a statistically significant result in acute PEF (Begg and Mazumdar $z=-2.04$, $P=0.042$ ). All other measures were either not statistically significant or could not be calculated because of the small number of publications associated with particular end points. We reason that the PEF result is not indicative of publication bias, per se, but rather a reflection of small sample size in the included studies.

\section{DISCUSSION}

\section{Acute effect}

The included literature suggests that abdominal FES can be used to achieve an acute improvement in CPF after SCI, whereas the acute effect of abdominal FES on $\mathrm{FEV}_{1}$ approached significance. Furthermore, it would appear that electrode position did not affect these changes, with Butler et al. ${ }^{2}$ and Gollee et al. ${ }^{3}$ finding similar increases 
a

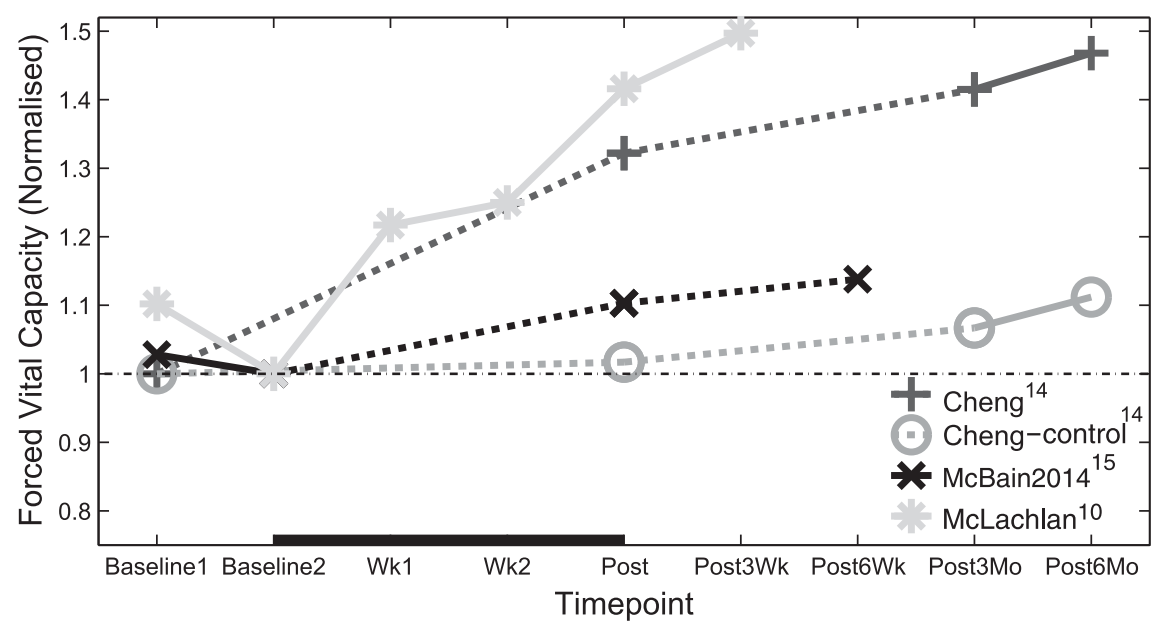

b

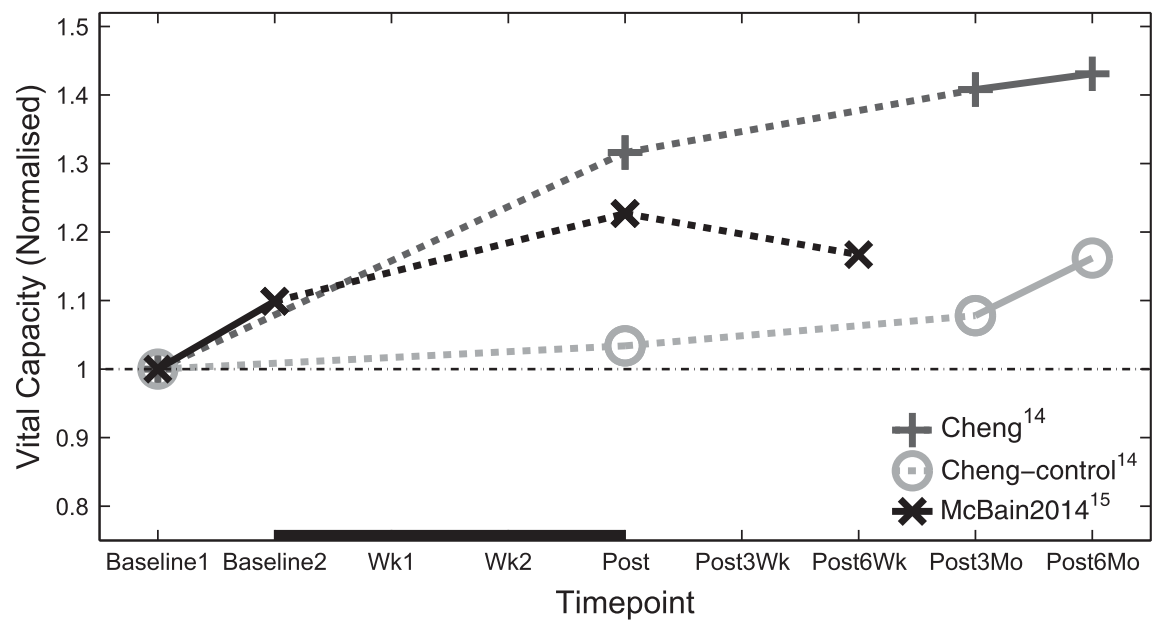

C

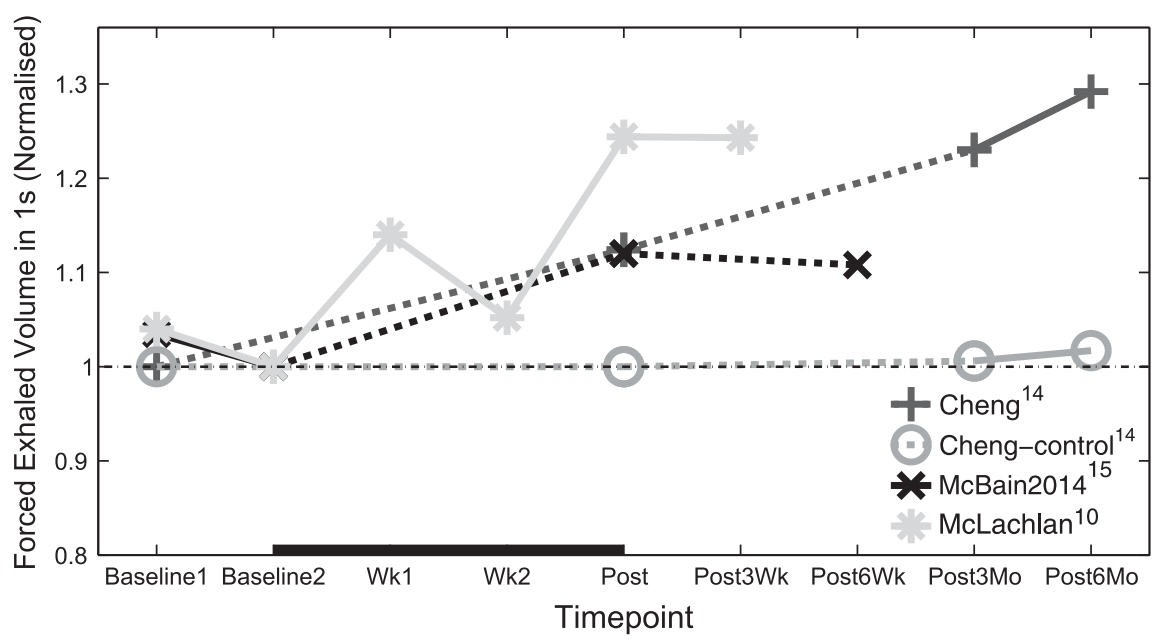

Figure 4 Effect of abdominal FES training on unassisted respiratory function. (a) Forced vital capacity, (b) vital capacity, (c) forced exhaled volume in $1 \mathrm{~s}$, (d) peak expiratory flow, (e) maximum expiratory pressure. Data are normalised based on minimum within-study values for each measure of respiratory function. Black bar along the bottom of the plot represents a period of abdominal FES training. Solid lines between time points indicate consecutive measurements; dotted lines indicate measurements that span $>1$ time point. Note: The time intervals between baseline 1 and baseline 2 , and baseline 2 and post-training, are not equal for each study.

in CPF (SMD 0.69 versus 0.68), despite Butler et al. using only four electrodes compared with Gollee et al.'s eight. McLachlan et al. ${ }^{10}$ and Langbein et al. ${ }^{5}$ found similar increases in $\mathrm{FEV}_{1}$ (SMD 0.17 versus 0.23), despite McLachlan et al. using eight electrodes to stimulate both the rectus abdominis muscles and the external oblique muscles, whereas Langbein et al. used eight electrodes to stimulate only the rectus abdominis muscles (Figure 2). However, low participant numbers and a lack of homogeneity across the included studies mean 

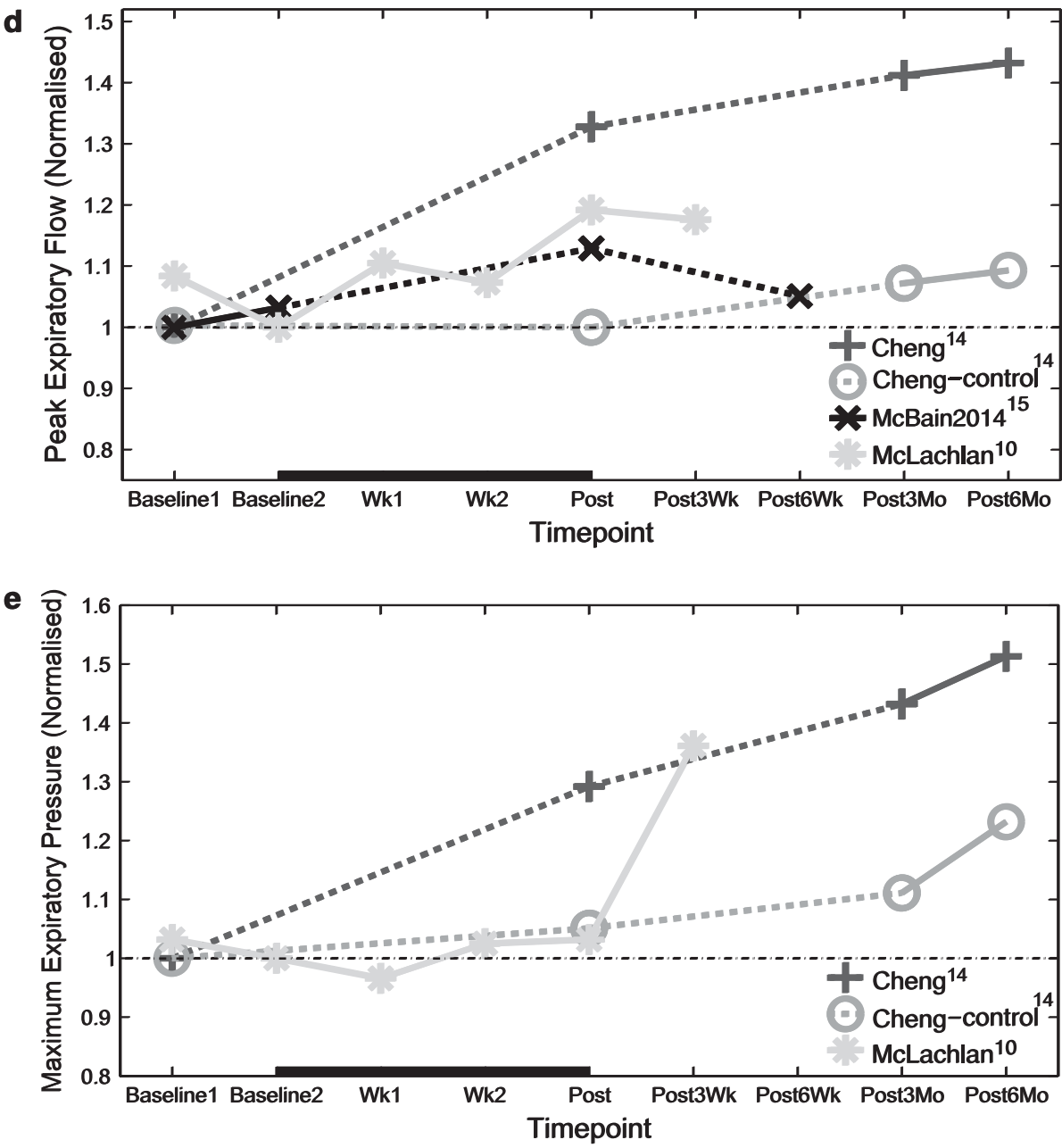

Figure 4 Continued.

Table 3 Comparison of respiratory function immediately and 6 months post abdominal FES training between intervention and control groups.

\begin{tabular}{|c|c|c|c|c|c|c|c|}
\hline Measure & Author & Modality & Participants & Time point & $S M D$ & $\mathrm{Cl}$ & P-value \\
\hline & Cheng 14 & Stim & 13 & 6 months post training & 1.04 & 0.166 to 1.914 & 0.020 \\
\hline & Cheng ${ }^{14}$ & Stim & 13 & 6 months post training & 0.683 & -0.133 to 1.499 & 0.101 \\
\hline \multirow[t]{2}{*}{$\mathrm{FEV}_{1}(\mathrm{I})$} & \multirow[t]{2}{*}{ Cheng ${ }^{14}$} & \multirow[t]{2}{*}{ Stim } & \multirow[t]{2}{*}{13} & Immediately post training & 0.371 & -0.412 to 1.154 & 0.353 \\
\hline & & & & 6 months post training & 0.052 & -0.717 to 0.822 & 0.894 \\
\hline \multirow[t]{2}{*}{$\operatorname{MEP}\left(\mathrm{cmH}_{2} \mathrm{O}\right)$} & \multirow[t]{2}{*}{ Cheng ${ }^{14}$} & \multirow[t]{2}{*}{ Stim } & \multirow[t]{2}{*}{13} & Immediately post training & 0.72 & -0.101 to 1.541 & 0.085 \\
\hline & & & & 6 months post training & 1.026 & 0.155 to 1.898 & 0.021 \\
\hline
\end{tabular}

Abbreviations: $\mathrm{Cl}$, confidence interval; $\mathrm{FEV}_{1}$, forced exhaled volume in $1 \mathrm{~s} ; \mathrm{FVC}$, forced vital capacity; MEP, maximum expiratory pressure; PEF, peak expiratory flow; SMS, standardised mean difference; $V_{c}$, vital capacity.

A positive SMD favours the intervention, while a negative SMD favours the control.

that these results must be interpreted with caution, with the low participant numbers meaning that individual studies have the potential to skew results. This problem is highlighted when interpreting the acute effect of abdominal FES on CPF, where despite three of the four included studies not finding a significant increase in this measure the large increase in $\mathrm{CPF}$ found by McBain et al. ${ }^{15}$ may be the sole reason for finding significance (Figure 3a). When investigating potential reasons for this difference, it was found that McBain et al. ${ }^{15}$ used the same electrode placement as Butler et al. ${ }^{2}$ (Figure 2) in addition to very similar patients and stimulation parameters (Table 1) and measurement techniques; yet, the results were markedly different (SMD 10.00 versus 0.69 ). This suggests that there were unidentified methodological differences that could have resulted in the variability between the studies. Because of the heterogeneity of SCI, it is possible 
that the study populations may vary between studies that are applying the same inclusion and exclusion criteria.

\section{Chronic effect}

When examining the chronic effect of abdominal FES training, the included literature suggests that abdominal FES training leads to an improvement in FVC, $\mathrm{V}_{\mathrm{C}}$ and PEF after SCI. However, the long-term effect of abdominal FES training remains to be established. Although a continued improvement in FVC and MEP after the cessation of training was observed in all studies that investigated these measures, the improvements in $\mathrm{V}_{\mathrm{C}}, \mathrm{FEV}_{1}$ and PEF observed by Cheng et al. ${ }^{14}$ after cessation of training appear to contradict the decreases in these measures observed by McLachlan et al. ${ }^{10}$ and McBain et al. ${ }^{15}$ Therefore, further work is needed to establish the long-term effect of abdominal FES training and whether any of the chronic improvements reported here are maintained past the end points of the included studies.

The aim of abdominal FES training is to reduce the likelihood of respiratory complications by preventing the build-up of secretions in the airways. In two separate publications, Bach et al. ${ }^{34}$ state that the minimum CPF needed to clear secretions is $2.67 \mathrm{ls}^{-1}$, and that patients with a CPF of $>4.51 \mathrm{~s}^{-1}$ are at less risk of developing acute respiratory failure. ${ }^{35}$ However, Cheng et al. ${ }^{14}$ were the only authors to directly investigate the use of abdominal FES to reduce respiratory complications, demonstrating a statistically significant decrease in respiratory complications in an intervention group that received 4 weeks of abdominal FES training. During the intervention period, the FVC, $\mathrm{FEV}_{1}$ and PEF of this intervention group increased by 0.55 , 0.22 and $0.971 \mathrm{~s}^{-1}$, respectively. Because of the reduction in respiratory complications associated with these improvements in respiratory function, these levels of improvement can be regarded as clinically significant and may be a beneficial benchmark for future abdominal FES studies.

\section{Physiology of abdominal FES}

$\mathrm{CPF}$ and $\mathrm{FEV}_{1}$ are forced expiratory manoeuvres and are both dependent on the intrathoracic pressure generated during exhalation. The acute effect of abdominal FES on CPF and $\mathrm{FEV}_{1}$ found in this review can therefore most likely be explained by the fact that abdominal FES has been shown to increase intrathoracic pressure in patients with SCI. ${ }^{2,36}$

The physiology of the chronic effect of abdominal FES is less well understood. Maximising FVC and PEF relies on generating the greatest MEP, which is dependent on expiratory muscle strength. As the thickness and hence strength of the abdominal muscles has been shown to be reduced in SCI, ${ }^{37}$ one explanation for the chronic increases in FVC and PEF reported here is that, even for patients with complete paralysis of the abdominal muscles, abdominal FES training thickens and strengthens the abdominal muscles, altering the passive biomedical properties of the respiratory system. ${ }^{10}$ Such strengthening of muscles after FES training has been demonstrated through a shift in the force frequency curve, ${ }^{38}$ increased muscle blood supply ${ }^{39}$ and muscle fibre-type conversion towards more fatigue-resistant fibres. ${ }^{39,40}$ McLachlan et al. ${ }^{10}$ propose that this strengthening of the abdominal muscles leads to greater support of the abdominal contents, which act as a fulcrum as the diaphragm contracts, placing the diaphragm in a more efficient mechanical position to expand the lower lung after abdominal FES training. There is also evidence in the literature to suggest that exercise contributes to an increase in brain-derived neurotrophic factor, which may promote synaptic and functional plasticity within the brain and spinal cord. ${ }^{41,42}$ However, currently postulation, such as corticospinal plasticity, may be the reason for the continued increase in respiratory function observed after the cessation of abdominal FES.

\section{Current limitations}

Although the main focus of the included studies was to improve respiratory function, the driver for such an improvement is improved sputum clearance and an associated reduction in respiratory complications. Cheng et al. ${ }^{14}$ were the only authors to investigate the impact of abdominal FES on the rates of respiratory complications. As respiratory complication rates are ultimately the primary clinical outcome measure to assess the efficacy of abdominal FES, future abdominal FES studies, particularly those that apply an abdominal FES training programme, should strive to investigate this effect.

Along with low participant numbers, a lack of standardised protocol was a significant burden to fully establishing the acute and chronic treatment effect of abdominal FES. For both study types, the range of stimulation devices, stimulation parameters and electrode positions used in the included studies is likely to have contributed to some of the variability observed in the meta-analysis. However, it is worth noting that McBain et al. ${ }^{43}$ have demonstrated that CPF plateaued when increasing stimulation intensity, suggesting that stimulation intensity alone may not be the only factor causing this variation. Only three of the included studies ${ }^{9,14,15}$ employed a random allocation of participants and only one study ${ }^{14}$ used control groups. In addition, this was the only study that blinded the assessor, and no studies blinded the participants to the intervention. This lack of blinding and randomisation may create a bias in the results, again making it difficult to fully ascertain the acute or chronic effect of abdominal FES.

\section{Future research}

To enable easier comparisons of studies, future abdominal FES studies should strive to use standardised protocols, particularly with regard to electrode placement, stimulation parameters, inclusion and exclusion criteria, and training period. Standardisation of these factors should remove a significant amount of the variability seen in the studies reported to date. Various solutions exist to facilitate such standardisation. First, McCaughey et al. ${ }^{44}$ propose the use of electrical stimulation to detect the motor points of the abdominal muscles, enabling standardisation of electrode placement. A number of techniques have also been proposed to standardise the muscle contraction achieved with abdominal FES. Butler et al. ${ }^{2}$ and McBain et al. ${ }^{15}$ increased stimulation intensity until a $P_{\mathrm{ga}}$ of $40 \mathrm{cmH}_{2} \mathrm{O}$ was achieved. Although this technique allows quantitative standardisation, it requires the use of an intrusive nasal catheter. Most studies included in this review adjusted stimulation parameters until a strong, even, visible contraction of the abdominal muscles was achieved. Although this may be less precise than the method used by Butler et al. and McBain et al., it should enable a consistent muscle contraction to be achieved in a non-intrusive manner. This review also identifies that abdominal FES can be used to improve the respiratory function of a wide range of SCI patients, ranging from ventilator-dependent tetraplegic patients ${ }^{31}$ to non-ventilator-dependent paraplegic patients, ${ }^{2,15}$ with no obvious exclusion criteria identified from either a safety or efficacy perspective. However, further studies are required to identify the patient groups that would benefit most from abdominal FES. Finally, the majority of abdominal FES training protocols have applied stimulation for 4 weeks, with varied follow-up times. ${ }^{9,10,14}$ Although all studies observed an improvement in respiratory function over this period, further study is required to ascertain the optimum abdominal FES training duration and to establish a standard follow-up time post intervention. 
Standardisation of the reporting of results is also required. A number of the included studies reported incomplete results, particularly pertaining to a lack of s.d.'s and confidence intervals, which meant that they could not be included in the analysis. Studies also employed a range of statistical analysis techniques, with different levels of statistical power. The correct use of standardised statistics would enable the greatest possibility of finding a treatment effect. Although not a panacea, a potential solution to the problem of the low participant numbers and the lack of standard protocol observed in the included studies is multi-centre RCTs, which use common protocols.

Although not included in the meta-analysis because of a lack of data, three studies have reported that Abdominal FES led to a significant acute increase in tidal volume $\left(V_{\mathrm{T}}\right)$ for participants with an $\mathrm{SCI}, 3,7,8$ indicating that this measure may be worthy of further exploration. Kandare et al. ${ }^{31}$ also demonstrated that abdominal FES could be used to support respiration for patients who lacked spontaneous ventilation, with the intervention able to provide an adequate $V_{\mathrm{T}}$ for up to $3.5 \mathrm{~min}$. This indicates that abdominal FES may be a useful short-term alternative or back up to mechanical ventilation. Although outside the scope of this review, two articles have also reported that abdominal FES can be used to assist ventilator weaning for patients with SCI. ${ }^{45,46}$ Further research may indicate that this intervention is suitable for other ventilator-dependent patient groups. Finally, Butler et al. ${ }^{2}$ and Lin et al. ${ }^{32}$ showed that combining abdominal FES with an abdominal binder led to an acute increase in $\mathrm{CPF}$, with Lin et al. finding that for 12 tetraplegic patients this increase was significantly greater than that achieved using the binder alone. A further meta-analysis is required to compare the increases in respiratory function achieved using either an abdominal binder or an abdominal FES.

As abdominal FES can be used to activate paralysed muscles, the majority of abdominal FES studies have focused on patients with SCI. Although the results with this patient group are promising, there are likely to be other patient groups who would benefit from abdominal FES. Ito et al. ${ }^{13}$ reported that abdominal FES could be used to achieve an acute improvement in tidal volume $V_{\mathrm{T}}$ for patients with chronic obstructive pulmonary disease. Jung et al. demonstrated that the use of abdominal FES training with patients with stroke could be used to achieve a significant increase in $\mathrm{FEV}_{1}$ and PEF compared with matched controls. ${ }^{11}$ Finally, $\mathrm{Na}$ et al. demonstrated that abdominal FES training could be used to achieve a statistically significant increase in FVC, $\mathrm{FEV}_{1}$ and $\mathrm{CPF}$ of patients with traumatic brain lesion, whereas no increase in these measures was seen in a matched control group that received no intervention. ${ }^{12}$ This indicates a potential new pathway for abdominal FES research, which may be applicable for a larger range of patient groups.

\section{CONCLUSION}

The included literature indicates that abdominal FES can improve respiratory function in an acute and long-term manner after SCI, with abdominal FES found to cause an acute improvement in CPF and abdominal FES training found to lead to a chronic increase in unassisted $\mathrm{V}_{\mathrm{C}}, \mathrm{FVC}$ and PEF. However, a lack of homogeneity across the studies and low participant numbers made it difficult to fully establish the treatment effect. Therefore, further randomised control trials that follow a standardised protocol and recruit larger numbers of participants are required to fully quantify the efficacy of abdominal FES.

\section{DATA ARCHIVING}

There were no data to deposit.

\section{CONFLICT OF INTEREST}

AJM is employed by Liberate Medical LLC, a medical device company that is developing an abdominal muscle stimulator. EJM and RF received financial support from Liberate Medical LLC to conduct this review.

1 Sheffler LR, Chae J. Neuromuscular electrical stimulation in neurorehabilitation. Muscle Nerve 2007; 35: 562-590.

2 Butler JE, Lim J, Gorman RB, Boswell-Ruys C, Saboisky JP, Lee BB et al. Posterolateral surface electrical stimulation of abdominal expiratory muscles to enhance cough in spinal cord injury. Neurorehabil Neural Repair 2011; 25: 158-167.

3 Gollee H, Hunt KJ, Allan DB, Fraser MH, McLean AN. A control system for automatic electrical stimulation of abdominal muscles to assist respiratory function in tetraplegia. Med Eng Phys 2007; 29: 799-807.

4 Jaeger RJ, Turba RM, Yarkony GM, Roth EJ. Cough in spinal cord injured patients: comparison of three methods to produce cough. Arch Phys Med Rehabil 1993; 74: 1358-1361.

5 Langbein WE, Maloney C, Kandare F, Stanic U, Nemchausky B, Jaeger RJ. Pulmonary function testing in spinal cord injury: effects of abdominal muscle stimulation. J Rehabil Res Dev 2001; 38: 591-597.

6 Linder SH. Functional electrical stimulation to enhance cough in quadriplegia. Chest 1993; 103: 166-169.

7 Sorli J, Kandare F, Jaeger RJ, Stanic U. Ventilatory assistance using electrical stimulation of abdominal muscles. IEEE Trans Rehabil Eng 1996; 4: 1-6.

8 Stanic U, Kandare F, Jaeger R, Sorli J. Functional electrical stimulation of abdominal muscles to augment tidal volume in spinal cord injury. IEEE Trans Rehabil Eng 2000; 8: 30-34.

9 Zupan A, Savrin R, Erjavec T, Kralj A, Karcnik T, Skorjanc T et al. Effects of respiratory muscle training and electrical stimulation of abdominal muscles on respiratory capabilities in tetraplegic patients. Spinal Cord 1997; 35: 540-545.

10 McLachlan AJ, McLean AN, Allan DB, Gollee H. Changes in pulmonary function measures following a passive abdominal functional electrical stimulation training program. J Spinal Cord Medicine 2013; 36: 97-103.

11 Jung JH, Shim JM, Kwon HY, Kim HR, Kim BI. Effects of abdominal stimulation during inspiratory muscle training on respiratory function of chronic stroke patients. J Phys Ther Sci 2014; 26: 73-76.

$12 \mathrm{Na}$ EH, Han SJ, Yoon TS. Effect of active pulmonary rehabilitation on pulmonary function in patients with brain lesion. NeuroRehabilitation 2014; 35: 459-466.

13 Ito K, Nozoe T, Okuda M, Nonaka K, Yamahara J, Horie J et al. Electrically stimulated ventilation feedback improves the ventilation pattern in patients with COPD. J Phys Ther Sci 2015; 27: 325-330.

14 Cheng PT, Chen CL, Wang CM, Chung CY. Effect of neuromuscular electrical stimulation on cough capacity and pulmonary function in patients with acute cervical cord injury. J Rehabil Med 2006; 38: 32-36.

15 McBain RA, Boswell-Ruys CL, Lee BB, Gandevia SC, Butler JE. Abdominal muscle training can enhance cough after spinal cord injury. Neurorehabil Neural Repair 2013; 27: 834-843.

16 Spungen AM, Bauman WA, Lesser M, McCool FD. Breathing pattern and ventilatory control in chronic tetraplegia. Lung 2009; 187: 375-381.

17 Jackson $A B$, Groomes TE. Incidence of respiratory complications following spinal cord injury. Arch Phys Med Rehabil 1994; 75: 270-275.

18 Cardenas DD, Hoffman JM, Kirshblum S, McKinley W. Etiology and incidence of rehospitalization after traumatic spinal cord injury: a multicenter analysis. Arch Phys Med Rehabil 2004; 85: 1757-1763.

19 Egger M, Smith GD, O'Rourke K. Rationale, potentials, and promise of systematic reviews. In: Egger M, Smith GD, Altman DG (eds). Systematic Reviews in Health Care: Meta-analysis in context, BMJ Publishing Group: London, UK. 2011.

20 Chhabra SK. Forced vital capacity, slow vital capacity, or inspiratory vital capacity: which is the best measure of vital capacity? J Asthma 1998; 35: 361-365.

21 DerSimonian R, Laird N. Meta-analysis in clinical trials. Control Clin Trials 1986; 7: 177-188.

22 Higgins JP, Thompson SG. Quantifying heterogeneity in a meta-analysis. Stat Med 2002; 21: 1539-1558.

23 Higgins JP, Thompson SG, Deeks JJ, Altman DG. Measuring inconsistency in meta-analyses. BMJ 2003; 327: 557-560.

24 Glass GV. Primary, secondary, and meta-analysis of research. Educ Res 1976; 5: 3-8.

25 Bluechardt MH, Wiens M, Thomas SG, Plyley MJ. Repeated measurements of pulmonary function following spinal cord injury. Paraplegia 1992; 30: 768-774.

26 Ledsome JR, Sharp JM. Pulmonary function in acute cervical cord injury. Am Rev Respiratory Dis 1981; 124: 41-44.

27 Begg CB, Mazumdar M. Operating characteristics of a rank correlation test for publication bias. Biometrics 1994; 50: 1088-1101.

28 Egger M, Davey Smith G, Schneider M, Minder C. Bias in meta-analysis detected by a simple, graphical test. BMJ 1997; 315: 629-634.

29 Harris RJ, Bradburn MJ, Deeks JJ, Harbord RM, Altman DG, Sterne JAC. metan: fixed- and random-effects meta-analysis. Stata J 2008; 8: 3-28.

30 Sterne JAC, Bradburn MJ, Egger M. Meta analysis in stata. In: Egger M, Smith GD, Altman DG (eds). Systematic Reviews in Health Care: Meta-Analysis in Context, 2nd edn, BMJ Publishing Group: London, UK. 2001, 347-369. 
31 Kandare F, Exner G, Jeraj J, Aliverti A, Dellacá R, Stanič U et al. Breathing induced by abdominal muscle stimulation in individuals without spontaneous ventilation. Neuromodulation 2002; 5: 180-185.

32 Lin $\mathrm{KH}$, Lai YL, Wu HD, Wang TQ, Wang YH. Effects of an abdominal binder and electrical stimulation on cough in patients with spinal cord injury. J Formos Med Assoc 1998; 97: 292-295.

33 Spivak E, Keren O, Niv D, Levental J, Steinberg F, Barak D et al. Electromyographic signal-activated functional electrical stimulation of abdominal muscles: the effect on pulmonary function in patients with tetraplegia. Spinal Cord 2007; 45: 491-495.

34 Bach JR, Saporito LR. Criteria for extubation and tracheostomy tube removal for patients with ventilatory failure. Chest 1996; 110: 1566-1571.

35 Bach JR, Ishikawa Y, Kim H. Prevention of pulmonary morbidity for patients with Duchenne muscular dystrophy. Chest 1997; 112: 1024-1028.

36 McBain RA, Boswell-Ruys CL, Lee BB, Gandevia SC, Butler JE. Electrical stimulation of abdominal muscles to produce cough in spinal cord injury: effect of stimulus intensity. Neurorehabil Neural Repair 2014; 29: 364-369.

37 Estenne M, Pinet C, De Troyer A. Abdominal muscle strength in patients with tetraplegia. Am J Res Crit Care Med 2000; 161 (3 Pt 1): 707-712.

38 Shields RK, Dudley-Javoroski S. Musculoskeletal plasticity after acute spinal cord injury: effects of long-term neuromuscular electrical stimulation training. J Neurophysiol 2006; 95: 2380-2390.

39 Gerrits HL, de Haan A, Sargeant AJ, Dallmeijer A, Hopman MT. Altered contractile properties of the quadriceps muscle in people with spinal cord injury following functional electrical stimulated cycle training. Spinal Cord 2000; 384: 214-223.
40 Dudley-Javoroski S, Shields RK. Muscle and bone plasticity after spinal cord injury: review of adaptations to disuse and to electrical muscle stimulation. J Rehabil Res Dev 2008; 45: 283-296.

41 Ragnarsson KT. Functional electrical stimulation after spinal cord injury: current use, therapeutic effects and future directions. Spinal Cord 2008; 46: 255-274.

42 Vaynman S, Gomez-Pinilla F. License to run: exercise impacts functional plasticity in the intact and injured central nervous system by using neurotrophins. Neurorehabil Neural Repair 2005; 19: 283-295.

43 McBain RA, Boswell-Ruys CL, Lee BB, Gandevia SC, Butler JE. Electrical stimulation of abdominal muscles to produce cough in spinal cord injury: effect of stimulus intensity. Neurorehabil Neural Repair 2015; 29: 362-369.

44 McCaughey EJ, McLean AN, Allan DB, Gollee H. Detection of the motor points of the abdominal muscles. Eur J Appl Phys 2014; 114: 2483-2489.

45 Lee BB, Boswell-Ruys C, Butler JE, Gandevia SC. Surface functional electrical stimulation of the abdominal muscles to enhance cough and assist tracheostomy decannulation after high-level spinal cord injury. J Spinal Cord Med 2008; 31. 78-82.

46 McCaughey EJ, Berry HR, McLean AN, Allan DB, Gollee H. Abdominal functional electrical stimulation to assist ventilator weaning in acute tetraplegia: a cohort study. PloS ONE 2015; 10: e0128589.

47 Kirshblum SC, Burns SP, Biering-Sorensen F, Donovan W, Graves DE, Jha A et al. International standards for neurological classification of spinal cord injury (revised 2011). J Spinal Cord Med 2011; 34: 535-546. 